\title{
Elemental abundances for HgMn stars observed with EBASIM echelle spectrograph at CASLEO
}

\author{
Olga I. Pintado ${ }^{1,2} \dagger$ and Saul J. Adelman ${ }^{3,4}$ \\ ${ }^{1}$ Consejo Nacional de Investigaciones Cientíificas y Técnicas, Argentina \\ ${ }^{2}$ Departamento de Física - FACET -Universidad Nacional de Tucumán, Argentina \\ email: opintado@tucbbs.com.ar \\ ${ }^{3}$ Department of Physics, The Citadel, 171 Moultrie Street, Charleston, SC 29409, USA \\ email: adelmans@citadel.edu \\ ${ }^{4}$ Guest Investigator, Dominion Astrophysical Observatory, Herzberg Institute of Astrophysics, \\ National Research Council of Canada, 5071 West Saanich Road, Victoria, BC V9E 2E7, \\ Canada
}

\begin{abstract}
Elemental abundance analysis are derived for the Mercury-Manganese stars HR 4817 (B8 II/III) and $\mu$ Lep (B9 IV) using CCD recorded exposures obtained at the EBASIM echelle spectrograph at the 2.1-m CASLEO (Complejo Astronómico El Leoncito) telescope in Argentina. The spectra coverage is $390-900 \mathrm{~nm}$. The results are compared with previous analyses made with spectra taken using the REOSC echelle spectrograph at CASLEO, the coudé feed telescope at Kitt Peak National Observatory, and/or with the Dominion Astrophysical Observatory coudé spectrograph. As these new spectra go farther into the red and have better resolution than those obtained with the REOSC, we could make better abundance determinations.
\end{abstract}

Keywords. Stars: abundances, individual:(HR 4817, $\mu$ Lep), stars: chemically peculiar stars

\section{Introduction}

We report observations made using the new EBASIM echelle spectrograph at the 2.1-m telescope of the CASLEO observatory of the Mercury-Manganese (HgMn) stars HR 4817 and $\mu$ Lep. A detailed description of the spectrograph is found in Pintado \& Adelman (2003). The spectra coverage is $390-900 \mathrm{~nm}$. We used two gratings as cross dispersors, one with $226 \mathrm{lmm}^{-1}$ and a $650 \mathrm{~nm}$ blaze angle, the other with $115 \mathrm{lmm}^{-1}$ and a 1100 $\mathrm{nm}$ blaze angle. The CCD detectors were from Tektroniks with 1024 x 1024 pixels (pixel size $=24 \mu \mathrm{m} \times 24 \mu \mathrm{m}$ ) and one designated as Roper with $1340 \times 1300$ pixels (pixel size $=$ $20 \mu \mathrm{m} \times 20 \mu \mathrm{m})$. Table 1 shows the observing log.

HR 4817 (= HD 110073) $\left(v \sin i=23 \mathrm{~km} \mathrm{~s}^{-1}\right)$ was studied by Adelman \& Philip (1994) and Adelman \& Pintado (1997). These investigators found $T_{\text {eff }}=12900 \mathrm{~K}, \log g=3.72$. $\mu$ Leporis $(=\mathrm{HD} 33904=\mathrm{HR} 1702)\left(v \sin i=18 \mathrm{~km} \mathrm{~s}^{-1}\right)$ was analyzed by Adelman (1987) and Adelman \& Pintado (1997). These authors derived $T_{\text {eff }}=12500 \mathrm{~K}$ and $\log g=3.5$.

$\dagger$ Visiting Astronomer at Complejo Astronómico El Leoncito operated under agreement between Consejo Nacional de Investigaciones Científicas y Técnicas de la República Argentina and the National Universities of La Plata, Córdoba and San Juan. 
Table 1. Observing Log

\begin{tabular}{lccccc}
\hline Star & Date & Wavelength range $(\mathrm{nm})$ & Grating & Detector & Number of spectra \\
\hline \multirow{2}{*}{ HR 4817 } & Jun. 2000 & $380-750$ & 226 & Tek & 4 \\
& Feb. 2001 & $380-750$ & 226 & Tek & 5 \\
& Apr. 2003 & $520-720$ & 226 & Roper & 2 \\
\multirow{4}{*}{ Lep } & Feb. 2001 & $640-890$ & 150 & Tek & 2 \\
& Oct. 2003 & $360-650$ & 226 & Roper & 2 \\
& Oct. 2003 & $640-890$ & 150 & Roper & 2 \\
\hline
\end{tabular}

\section{Data analyses}

The EBASIM spectral reductions were made with IRAF 2.12. Using bias, darks and flat fields we obtain a combined flat field which was used to divide the specta to remove pixelto-pixel variatons. The spectra with the same wavelength range were combined using SCOMBINE. The extraction was performed with APALL and the wavelength calibration with ECIDENTIFY and DISPCOR, using the exposures of a Th-Ar comparison lamp. The spectra were rectified with REDUCE and measured with VLINE (Hill \& Fisher 1986).

ATLAS9 model atmospheres and WIDTH9 (Kurucz 1993) were used in the analyses. The results for the HgMn stars are compared with previous analyses made with spectra taken using the REOSC echelle spectrograph at CASLEO, the coudé feed telescope at Kitt Peak National Observatory, and/or with the Dominion Astrophysical Observatory's (DAO) coudé spectrograph in Table 2. As these new spectra go farther into the red and have better resolution than those obtained with the REOSC, we could make better determinations of abundances. The signal-to-noise ratio $(S / N)$ is typically $400-500$ in the center of the orders, but only 50-200 at the ends of the orders.

Table 2. Comparison of abundances

\begin{tabular}{lcccccc}
\hline & EBASIM & REOSC $^{1}$ & KITT PEAK $^{2}$ & EBASIM & REOSC $^{1}$ & DAO $^{3}$ \\
\hline Species & HR 4817 & HR 4817 & HR 4817 & $\mu$ Lep & $\mu$ Lep & $\mu$ Lep \\
\hline C II & -3.82 & -3.84 & -4.03 & -3.70 & -3.68 & -3.80 \\
O I & -3.00 & -3.03 & $\ldots$ & $\ldots$ & $\ldots$ & $\ldots$ \\
Mg I & -4.45 & -4.51 & $\ldots$ & -5.00 & -5.17 & -4.07 \\
Mg II & -4.80 & -4.76 & -4.89 & -4.80 & -4.76 & -4.77 \\
Si II & -4.70 & -4.76 & -4.80 & -4.25 & -4.29 & -4.33 \\
Si III & -4.90 & -4.86 & -4.96 & -4.30 & -4.22 & -4.46 \\
S II & -5.00 & -5.06 & -5.13 & -4.72 & -4.68 & -4.81 \\
Ca II & -5.43 & -5.36 & -5.43 & -5.30 & -5.27 & -5.24 \\
Ti II & -5.48 & -5.50 & -5.62 & -6.30 & -6.41 & -6.15 \\
Cr II & -5.45 & -5.40 & -5.37 & -5.90 & -5.89 & -6.03 \\
Mn I & -4.00 & -3.97 & -4.00 & -4.62 & -4.58 & -4.40 \\
Mn II & -4.10 & -4.04 & -4.27 & -4.50 & -4.45 & -4.50 \\
Fe II & -4.90 & -4.87 & -5.00 & -4.60 & -4.62 & -4.75 \\
Hg I & $\ldots$ & $\ldots$ & $\ldots$ & -5.70 & -5.73 & -5.72 \\
Hg II & $\ldots$ & $\ldots$ & $\ldots$ & -5.65 & -5.65 & -5.73 \\
\hline
\end{tabular}

1. Adelman, S.J \& Pintado, O.I., 1997, A\&AS, 125, 219,

2. Adelman, S.J. \& Philip, A G. D., 1994, PASP, 106, 1239,

3. Adelman, S.J., 1987, MNRAS, 228, 573. 


\section{Final comments}

Compared with our previous spectra obtained with the REOSC spectrograph, properly exposed spectra obtained using the EBASIM spectrograph had greater resolution and higher signal-to-noise ratios. This greater resolution allowed us to deconvolve blends which resulted in more consistent elemental abundances. A new CCD, designed Roper, has a slightly better quantum efficiency than the previous CCD in the red and considerably better in the blue. Even so this new detector permitted us to improve our detection of chemical elements with lines in whose wavelengths were greater than $700 \mathrm{~nm}$.

\section{Acknowledgements}

The authors thanks the Scientific Committee of CASLEO for the telescope time. SJA thanks The Citadel Foundation for their support and OIP thanks the CONICET and the ANPCyT for their support. OIP is grateful of the staff of CASLEO for their assistance during the observing runs. The authors acknowledge used of the Tek CCD supported by US NSF Grant AST 90-15827 to R. M. Rich.

\section{References}

Adelman, S.J. 1987, MNRAS 228, 573

Adelman, S.J. \& Philip, A. G. D. 1994, PASP 106, 1239

Adelman, S.J \& Pintado, O.I. 1997, A\& $A S$ 125, 219

Hill, G. \& Fisher, W.A. 1986, Publ. Dom. Astrophys. Obs. Victoria, 16, 27

Kurucz, R.L. 1993, ATLAS9 Stellar Atmosphere Programs and $2 \mathrm{~km} / \mathrm{s}$ grid, Kurucz CD-Rom, No 13, SAO, Cambridge, MA

Pintado, O.I. \& Adelman, S.J. 2003, A\&广A 406, 897 